Proceedings of the Edinburgh Mathematical Society (2002) 45, 257-276 (C)

DOI:10.1017/S0013091501000360 Printed in the United Kingdom

\title{
ANALYSIS OF OPTICAL TOMOGRAPHY WITH NON-SCATTERING REGIONS
}

\author{
NUUTTI HYVÖNEN \\ Helsinki University of Technology, Institute of Mathematics, \\ PO Box 1100, FIN-02015 HUT, Finland
}

(Received 26 March 2001)

\begin{abstract}
This paper provides mathematical analysis of optical tomography in a situation when the examined object, for example the human brain, is strongly scattering with non-scattering inclusions. Light propagation in biological tissue is often modelled by the diffusion approximation of the radiative transfer equation. To be justified, the diffusion approximation demands that the medium is strongly scattering. Naturally, this is not true for non-scattering inclusions, for which some other model is needed. This is found through geometrical optics. Combination of the two models leads to an elliptic partial differential equation with boundary conditions on the outer boundary as well as on the boundaries of the non-scattering regions. The well-posedness of this forward problem is the main concern of this work.
\end{abstract}

Keywords: optical tomography; non-scattering regions; radiative transfer equation; diffusion approximation; inverse problems

AMS 2000 Mathematics subject classification: Primary 35Q60; 35R30

\section{Introduction}

Over the past decade or so the scientific world has shown a growing interest in the research field known as biomedical optics. The development of a diagnostic imaging modality based on near-infrared (NIR) radiation has been considered to be one of the most important goals, and probably to be one of the most challenging. The progress towards optical tomography has, however, suffered from the lack of suitable instrumentation and an inadequate analytical treatment of the reconstruction problem. The aim of this work is to help solve the latter difficulty by modelling optical tomography mathematically in the presence of weakly scattering regions.

NIR tomography research has focused on a few possible low-cost clinical applications. The most important lines of research are, arguably, screening for breast cancer and the development of a cerebral imaging modality for mapping structure and function in newborn infants, and possibly adults too. The latter imaging problem is, in a way, the more difficult one, since it involves stronger absorption and scattering [1]. Further, the job is not made any easier by the non-scattering inclusions filled with cerebrospinal fluid - the main concern of this text. 
In optical tomography a physical body is illuminated with a flux of NIR photons and the outcoming flux is measured on the surface of the body. The idea is to reconstruct the optical properties inside the body by using the measured pairs of input and output fluxes. In order to have any success in the ill-posed reconstruction process, an appropriate model of photon transport inside the body is needed: the model should be in accordance with the experimental results and it should lead to a well-posed direct problem.

The models of photon transport used when modelling the optical imaging problem are usually derived under the assumption that the medium in question is strongly scattering, which means that for the medium, scattering is more characteristic than absorption. This kind of assumption is valid for many tissues [1] , for example the human breast and brain, but not at all for cavities filled with weakly scattering spinal fluid. Actually, all attempts to solve the inverse problem for the optical properties inside the examined object are doomed if no alternative model of photon transport is used for weakly scattering regions [5].

In this text we examine the radiosity-diffusion model [5], which is a combination of the diffusion approximation of the radiative transfer equation for strong scattering and geometrical optics for non-scattering regions. The aim is to prove that this model induces a well-defined direct problem. For other aspects of optical tomography, such as reconstruction methods and technical, physical and medical details, we refer to the excellent papers $[\mathbf{1}],[\mathbf{7}],[\mathbf{2}]$ and $[\mathbf{5}]$.

The text is organized as follows. Section 2 introduces the physical concepts, the radiosity-diffusion model and formulates the classical direct problem. In $\S 3$ we examine with care an integral operator that is needed for sewing together the strongly and non-scattering regions. The mathematical bases of this text can be found in this section. Finally, $\S 4$ formulates the weak direct problem and proves the existence of a unique solution.

\section{Approximating light propagation}

Propagation of electromagnetic radiation in a medium is governed by Maxwell's equations. Particularly, this holds for the case of interest here: namely, NIR light travelling through some biological tissue. However, since the wavelength of NIR light is small compared with the characteristic distances of human tissue, the exact models are totally useless for us. Therefore, we will model light propagation by using the radiative transfer equation, also known as the Boltzmann equation, and its diffusion approximation. We will handle the strongly scattering and the non-scattering regions separately.

\subsection{The radiative transfer equation}

Let $\Omega \subset \mathbb{R}^{n}, n=2$ or $n=3$, be a bounded body with a $C^{2}$-boundary and connected complement. We consider radiation in the body $\Omega$. The first concept we introduce is the radiation flux density. Let $\hat{\theta} \in S^{n-1}$ be a direction vector. The radiation flux density at $x \in \Omega$ at time $t \in \mathbb{R}$ through the infinitesimal solid angle $\mathrm{d} s$ in direction $\hat{\theta}$ is written as

$$
\mathrm{d} \boldsymbol{J}(x, t, \hat{\theta})=I(x, t, \hat{\theta}) \hat{\theta} \mathrm{d} s(\hat{\theta}),
$$


where the amplitude $I(x, t, \hat{\theta})$ is called the radiance. In the framework of the transport theory, this scalar function satisfies the radiative transfer equation

$$
\begin{aligned}
\frac{1}{c} I_{t}(x, t, \hat{\theta})+\hat{\theta} \cdot \nabla I(x, t, \hat{\theta}) & +\left(\mu_{\mathrm{a}}(x)+\mu_{\mathrm{s}}(x)\right) I(x, t, \hat{\theta}) \\
& -\mu_{\mathrm{s}}(x) \int_{S^{n-1}} f(x, \hat{\theta}, \hat{\omega}) I(x, t, \hat{\omega}) \mathrm{d} s(\hat{\omega})=q(x, t, \hat{\theta}),
\end{aligned}
$$

where $c$ is the speed of light (assumed to be constant), the positive scalar functions $\mu_{\mathrm{a}}$ and $\mu_{\mathrm{s}}$ are the scattering and absorption coefficients, respectively, and $q$ denotes the source term, which is assumed to vanish in this discussion. The kernel $f$ is the scattering phase function, satisfying the following three conditions:

$$
\begin{gathered}
\int_{S^{n-1}} f(x, \hat{\theta}, \hat{\omega}) \mathrm{d} s(\hat{\theta})=\int_{S^{n-1}} f(x, \hat{\theta}, \hat{\omega}) \mathrm{d} s(\hat{\omega})=1, \\
f(x, \hat{\theta}, \hat{\omega}) \geqslant 0, \quad x \in \mathbb{R}^{n}, \quad \hat{\theta}, \hat{\omega} \in S^{n-1} \\
f(x, \hat{\theta}, \hat{\omega})=f(x,-\hat{\omega},-\hat{\theta}), \quad \hat{\theta}, \hat{\omega} \in S^{n-1} .
\end{gathered}
$$

Note that due to (2.2) and (2.3), for fixed $x, f$ may be regarded as a probability distribution on $S^{n-1}$ with respect to either of the variables $\hat{\theta}$ and $\hat{\omega}$. For more transport theory consult, for example, [3].

To end this subsection, we introduce some concepts that turn out to be useful to us later. Given the radiation flux density, the flux through an infinitesimal oriented surface patch $\hat{n} \mathrm{~d} S$ is obtained by integrating the flux density over all radiation directions:

$$
\begin{aligned}
\mathrm{d} \Phi(x, t) & =\left(\int_{S^{n-1}} \mathrm{~d} \boldsymbol{J}(x, t, \hat{\theta})\right) \cdot \hat{n} \mathrm{~d} S \\
& =\left(\int_{S^{n-1}} I(x, t, \hat{\theta}) \hat{\theta} \mathrm{d} s(\hat{\theta})\right) \cdot \hat{n} \mathrm{~d} S \\
& =\boldsymbol{J}(x, t) \cdot \hat{n} \mathrm{~d} S,
\end{aligned}
$$

where the vector field $\boldsymbol{J}$ is the energy current density. We also define the scalar function

$$
\varphi(x, t)=\int_{S^{n-1}} I(x, t, \hat{\theta}) \mathrm{d} s(\hat{\theta})
$$

called the energy fluency, which will turn out to be our most important playmate.

\subsection{Strong scattering}

Being an integrodifferential equation, the radiative transfer equation, as discussed above, leads easily to numerical problems of prohibitive size if no simplifications are made. The commonly used simplification is called the diffusion approximation, which has been shown to be justified for materials that are much more scattering than absorbing [5]-brain tissue for example.

We begin by introducing a projection operator that forms the heart of the diffusion approximation. 
Definition 2.1. Let $\hat{\theta}=\left(\theta_{1}, \ldots, \theta_{n}\right) \in S^{n-1}$ be our variable and let

$$
H_{1}=\operatorname{span}\left\{1, \theta_{1}, \ldots, \theta_{n}\right\} \subset L^{2}\left(S^{n-1}\right) .
$$

We define $P$ to be the orthogonal projection mapping $L^{2}\left(S^{n-1}\right)$ onto $H_{1}$.

Note that the definition above makes sense since $H_{1}$ is clearly a closed subspace of $L^{2}\left(S^{n-1}\right)$, and thus the orthogonal projection is well defined [12]. Definition 2.1 is usually extended by letting $P_{n}$ be the orthogonal projection operator that maps $L^{2}\left(S^{n-1}\right)$ onto spherical harmonics of order $n$; since linear polynomials are always harmonic, $P_{1}=P$.

Next we write the radiative transfer equation in a simpler form

$$
\mathcal{B} I=0,
$$

where $\mathcal{B}$ is the linear integrodifferential operator induced by (2.1), and define what we mean by the diffusion approximation as follows.

Definition 2.2. The diffusion approximation of the radiative transfer equation (2.1) is defined as

$$
P \mathcal{B} P I=0 .
$$

The diffusion approximation, as it is given in Definition 2.2, is of course very abstract. With a little work [8], however, equation (2.6) can be written out explicitly. The following lemma presents the resulting equation.

Lemma 2.3. The explicit form of the diffusion approximation is

$$
\left(\frac{1}{c} \varphi_{t}+\mu_{\mathrm{a}} \varphi+\nabla \cdot \boldsymbol{J}\right)+n\left(\frac{1}{c} \boldsymbol{J}_{t}+\frac{1}{n} \nabla \varphi+\left(\mu_{\mathrm{a}}+\mu_{\mathrm{s}}(I-B)\right) \boldsymbol{J}\right) \cdot \hat{\theta}=0,
$$

where $I \in \mathbb{R}^{n \times n}$ is the identity matrix and $B(x) \in \mathbb{R}^{n \times n}$ is given by components as

$$
B_{j k}=\frac{n}{\left|S^{n-1}\right|} \int_{S^{n-1}} \int_{S^{n-1}} \theta_{j} \omega_{k} f(x, \hat{\theta}, \hat{\omega}) \mathrm{d} s(\hat{\theta}) \mathrm{d} s(\hat{\omega}) .
$$

In order to handle equation (2.7) efficiently, some information on matrix $B$ is needed. Hence we state a lemma that is a straightforward consequence of properties (2.2), (2.3) and (2.4).

Lemma 2.4. The real matrix $B(x) \in \mathbb{R}^{n \times n}$, introduced in Lemma 2.3, is symmetric and its spectrum is a subset of $[-1,1] \subset \mathbb{R}$. Particularly, it holds that

$$
|B(x)| \leqslant 1, \quad x \in \Omega .
$$

From Lemma 2.3, one sees that the diffusion approximation leads to a coupled system of equations, one being the part independent of $\hat{\theta}$, the other one being the multiplier of $\hat{\theta}$. In effect, $\varphi$ and $\boldsymbol{J}$ should satisfy the system

$$
\begin{aligned}
& \frac{1}{c} \varphi_{t}=-\nabla \cdot \boldsymbol{J}-\mu_{\mathrm{a}} \varphi, \\
& \frac{1}{c} \boldsymbol{J}_{t}=-\frac{1}{n} \nabla \varphi-\left(\mu_{\mathrm{a}}+(I-B) \mu_{\mathrm{s}}\right) \boldsymbol{J} .
\end{aligned}
$$


In this text we are interested in the case where the input field is modulated with a fixed harmonic frequency, i.e.

$$
I(x, t, \hat{\theta})=\mathrm{e}^{-\mathrm{i} \omega t} \hat{I}(x, \hat{\theta}) .
$$

Denoting the wavenumber by $k=\omega / c$, the diffusion approximation yields a new pair of equations

$$
\begin{aligned}
\mathrm{i} k \varphi & =\nabla \cdot \boldsymbol{J}+\mu_{\mathrm{a}} \varphi, \\
\mathrm{i} k \boldsymbol{J} & =\frac{1}{n} \nabla \varphi+\left(\mu_{\mathrm{a}}+(I-B) \mu_{\mathrm{s}}\right) \boldsymbol{J},
\end{aligned}
$$

where, for simplicity, we have denoted the time-harmonic amplitudes of $\varphi$ and $\boldsymbol{J}$ by the same symbols. By solving equation (2.10) for $\boldsymbol{J}$ and substituting into (2.9), we get the equation

$$
\nabla \cdot K \nabla \varphi+\left(\mathrm{i} k-\mu_{\mathrm{a}}\right) \varphi=0
$$

where

$$
K=(1 / n)\left(-\mathrm{i} k I+\mu_{\mathrm{a}} I+(I-B) \mu_{\mathrm{s}}\right)^{-1} .
$$

It turns out that $K$ really exists if some physically reasonable conditions are posed on the coefficients $\mu_{\mathrm{a}}$ and $\mu_{\mathrm{s}}$. The properties of the matrix $K$ are discussed in more detail in $\S 4$.

Next we aim to describe the boundary conditions of the regions with strong scattering. Consider a diffusive region $\Omega_{s} \subset \Omega$ with a $C^{2}$-boundary $\partial \Omega_{s}$. We will denote the exterior unit normal vector at $x \in \partial \Omega_{s}$ by $\hat{n}(x)$. The following simple lemma gives us all the information we need. The proof is again omitted [8].

Lemma 2.5. Within the diffusion approximation, the total flux inwards $(-)$ and outwards (+) at $x \in \partial \Omega_{\mathrm{s}}$ is given by

$$
\Phi_{ \pm}= \pm \gamma \varphi+\frac{1}{2} \hat{n} \cdot \boldsymbol{J}
$$

where the dimension-dependent constant $\gamma$ is

$$
\gamma=\frac{\Gamma(n / 2)}{\sqrt{\pi}(n-1) \Gamma((n-1) / 2)} .
$$

In particular, in dimensions $n=2,3$ we have

$$
\gamma_{2}=1 / \pi, \quad \gamma_{3}=\frac{1}{4} .
$$

The expression for the fluxes $\Phi_{ \pm}$above differs slightly from the one given in most references. Because the diffusion approximation is only an approximation, one cannot really say which convention is the correct one. The expression given in Lemma 2.5 is, however, carefully conducted from the mathematical model described by Definition 2.2 [8], and so it is one possible choice.

Denoting once again the time-harmonic amplitudes by the same symbols and solving (2.10) for $\boldsymbol{J}$, we conclude that for the time-harmonic case the boundary conditions read

$$
\Phi_{ \pm}= \pm \gamma \varphi-\frac{1}{2} \hat{n} \cdot K \nabla \varphi
$$

where the matrix $K$ is defined by (2.12). 


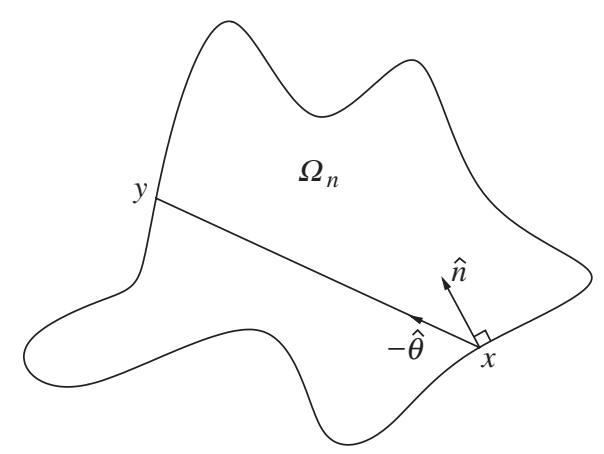

Figure 1. Light propagation in $\Omega_{n}$.

\subsection{Non-scattering regions}

In weakly scattering regions the diffusion approximation is no more valid [5] and so we will have to come up with something new. Let $\Omega_{n} \subset \Omega$ be a non-scattering region with a $C^{2}$-boundary. Because in a non-scattering region all the radiation is in the forward direction, we easily see that in $\Omega_{n}$ equation (2.1) yields the relation

$$
(1 / c) I_{t}(x, t, \hat{\theta})+\hat{\theta} \cdot \nabla I(x, t, \hat{\theta})+\tilde{\mu}_{\mathrm{a}} I(x, t, \hat{\theta})=0,
$$

where $\tilde{\mu}_{\mathrm{a}}$ is the absorption coefficient that is assumed to be constant in $\Omega_{n}$. Since we are still interested mainly in the time-harmonic case, $I(x, t, \hat{\theta})=\hat{I}(x, \hat{\theta}) \mathrm{e}^{-\mathrm{i} w t}$, we have

$$
\left(\tilde{\mu}_{\mathrm{a}}-\mathrm{i} k\right) \hat{I}+\hat{\theta} \cdot \nabla \hat{I}=0
$$

yielding an attenuated plane wave solution

$$
\hat{I} \sim \mathrm{e}^{-\left(\tilde{\mu}_{\mathrm{a}}-\mathrm{i} k\right) \hat{\theta} \cdot x} .
$$

Figure 1 illustrates the situation. Let $x \in \partial \Omega_{n}$ be a boundary point of the nonscattering region. We are looking for an approximate expression for the photon flux from $\Omega_{n}$ at $x$. Let us denote by $\hat{n}=\hat{n}(x)$ the unit normal vector of $\partial \Omega_{n}$ pointing into $\Omega_{n}$, and let $\hat{\theta}$ denote a direction vector with $\hat{\theta} \cdot \hat{n}(x)<0$. Consider the photon flux at $x$ coming from the direction $-\hat{\theta}$. Let $y(\hat{\theta}) \in \partial \Omega_{n}$ denote the first boundary point where the line emanating from $x$ into direction $-\hat{\theta}$ hits the boundary $\partial \Omega_{n}$. Since the radiation propagates with no scattering, we know that the radiation from this direction originates from $y(\hat{\theta})$. Hence, the contribution to the radiation flux density at $x$, originating from direction $-\hat{\theta}$, is

$$
\mathrm{d} \boldsymbol{J}(x, \hat{\theta})=\hat{\theta} \hat{I}(y, \hat{\theta}) \mathrm{e}^{-\left(\tilde{\mu}_{\mathrm{a}}-\mathrm{i} k\right) \hat{\theta} \cdot(x-y)} \mathrm{d} s(\hat{\theta}) .
$$

In accordance with (2.5), at $x$ the total flux out of the non-scattering region is obtained as an integral

$$
\Phi_{-}(x)=\int_{\{\hat{\theta} \cdot \hat{n}(x)<0\}} \hat{n}(x) \cdot \mathrm{d} \boldsymbol{J}(x, \hat{\theta}) .
$$


We want to express this formula as an integral over the boundary $\partial \Omega_{n}$. By elementary geometry, we observe that the surface element $\mathrm{d} S(y)$ of $\partial \Omega_{n}$ and the element $\mathrm{d} s(\hat{\theta})$ of the unit sphere are related through

$$
\hat{\theta} \cdot \hat{n}(y) \mathrm{d} S(y)=|x-y|^{n-1} \mathrm{~d} s(\hat{\theta}), \quad \hat{\theta}=\frac{x-y}{|x-y|} .
$$

To relate the value $\hat{I}(y, \hat{\theta})$ to the flux quantities, we assume that the total flux into the non-scattering region distributes uniformly to all directions, i.e. $\hat{I}(y, \hat{\theta})=\hat{I}_{0}(y)$ does not depend on $\hat{\theta}$; this kind of assumption seems pretty reasonable when one observes it from the diffusion approximation's point of view. Thus,

$$
\begin{aligned}
\Phi_{+}(y) & =\int_{\{\hat{\theta} \cdot \hat{n}(y)>0\}}(\hat{n}(y) \cdot \hat{\theta}) \hat{I}_{0}(y) \mathrm{d} s(\hat{\theta}) \\
& =\frac{\left|S^{n-2}\right|}{n-1} \hat{I}_{0}(y) .
\end{aligned}
$$

Substituting the obtained results, we get the formula

$$
\begin{aligned}
\Phi_{-}(x) & =\frac{n-1}{\left|S^{n-2}\right|} \int_{\partial \Omega_{n}} v(x, y) \frac{(\hat{n}(x) \cdot(x-y))(\hat{n}(y) \cdot(x-y))}{|x-y|^{n+1}} \mathrm{e}^{-\left(\tilde{\mu}_{\mathrm{a}}-\mathrm{i} k\right)|x-y|} \Phi_{+}(y) \mathrm{d} S(y) \\
& =\mathcal{G} \Phi_{+}(x)
\end{aligned}
$$

where $v(x, y)$ is a visibility function,

$$
v(x, y)= \begin{cases}1, & \text { if } t x+(1-t) y \in \Omega_{n} \text { for } 0<t<1 \\ 0, & \text { otherwise }\end{cases}
$$

Thus we have obtained a relation between inward and outward fluxes on the boundary of the non-scattering region, which is exactly what we were looking for.

\subsection{Formulation of the direct problem}

We have now gathered enough information on the strong scattering, the non-scattering regions and the boundary conditions to be able to formulate the direct problem in the strongly scattering region. Let us assume that our open domain $\Omega$ consists of the open non-scattering region $\Omega_{n}$ and the strongly scattering region $\Omega_{\mathrm{s}}=\Omega \backslash \bar{\Omega}_{n}$. All the boundaries are assumed to be of the class $C^{2}$ and $\Omega_{n}$ is assumed to lie strictly inside $\Omega$, meaning that $\operatorname{dist}\left(\partial \Omega_{n}, \partial \Omega\right)>0$. It is also assumed that no reflection of light occurs on the boundaries $\partial \Omega$ and $\partial \Omega_{n}$. The purpose of this last restriction is to keep things simple in order to be able to concentrate on the more important questions. It should not be too difficult, however, to extend the results of this work to situations in which reflection of light is allowed. 
From now on we will consider only the time-harmonic case. Let $\Phi_{\text {in }}$ denote the known input flux from the outside source on the boundary $\partial \Omega$. According to equation (2.15), in the framework of the diffusion approximation, the time-harmonic amplitude of the energy fluency $\varphi$ must satisfy the 'outer' boundary condition

$$
\Phi_{\text {in }}=-\left.\left(\gamma \varphi+\frac{1}{2} \hat{n} \cdot K \nabla \varphi\right)\right|_{\partial \Omega}
$$

On the other hand, we know that equation (2.15) is also valid on the boundary $\partial \Omega_{n}$. Thus, using relation (2.16), we get

$$
-\left.\left(\gamma \varphi+\frac{1}{2} \hat{n} \cdot K \nabla \varphi\right)\right|_{\partial \Omega_{n}}=\left.\mathcal{G}\left(\gamma \varphi-\frac{1}{2} \hat{n} \cdot K \nabla \varphi\right)\right|_{\partial \Omega_{n}},
$$

which is equivalent to the 'inner' boundary condition

$$
\left.\varphi\right|_{\partial \Omega_{n}}=-\left.\frac{1}{2 \gamma}(I+\mathcal{G})^{-1}(I-\mathcal{G})(\hat{n} \cdot K \nabla \varphi)\right|_{\partial \Omega_{n}} .
$$

We may now state the forward problem as follows.

Definition 2.6. Let $\Omega$ and $\Omega_{n}$ be as described above. The forward problem in the strongly scattering region is to find a function $\varphi$ satisfying

$$
\nabla \cdot K \nabla \varphi+\left(\mathrm{i} k-\mu_{\mathrm{a}}\right) \varphi=0
$$

in $\Omega \backslash \bar{\Omega}_{n}$, with the boundary conditions

$$
\Phi_{\text {in }}=-\left.\left(\gamma \varphi+\frac{1}{2} \hat{n} \cdot K \nabla \varphi\right)\right|_{\partial \Omega}
$$

and

$$
\left.\varphi\right|_{\partial \Omega_{n}}=-\left.\frac{1}{2 \gamma}(I+\mathcal{G})^{-1}(I-\mathcal{G})(\hat{n} \cdot K \nabla \varphi)\right|_{\partial \Omega_{n}} .
$$

The well-posedness of this problem is the main concern of our work. It turns out that under physically reasonable conditions the weak problem corresponding to the classical one introduced in Definition 2.6 has a unique solution. However, before the weak forward problem can be formulated and the unique existence of the solution can be proved, we need to know more about the behaviour of the boundary integral operator $\mathcal{G}$.

\section{Operators $\mathcal{G}$ and $I \pm \mathcal{G}$}

Our goal in the next two sections is to formulate the weak problem corresponding to the direct problem introduced in Definition 2.6 and to prove that there exists a unique solution for it. However, since we have too little information about the operator $\mathcal{G}$, we are forced to leave the actual playing with the weak problem to the next section and to concentrate now on the integral operators in hand. Sometimes, for the sake of motivation, it would be advisable, however, to sneak ahead and have a look at the stuff in $\S 4$. 


\subsection{On the operator $\mathcal{G}$}

We start this section by making our notation a little simpler.

Definition 3.1. We define kernels $h_{0}(x, y)$ and $h(x, y)$ as follows:

$$
h_{0}(x, y)=\frac{n-1}{\left|S^{n-2}\right|} v(x, y) \frac{(\hat{n}(x) \cdot(x-y))(\hat{n}(y) \cdot(x-y))}{|x-y|^{n+1}},
$$

where $v(x, y)$ is the visibility function given in (2.17), and

$$
h(x, y)=h_{0}(x, y) \mathrm{e}^{-\left(\tilde{\mu}_{\mathrm{a}}-\mathrm{i} k\right)|x-y|} .
$$

Next we want to say something about the function spaces on which $\mathcal{G}$ operates. The following lemma gives us useful and intuitively acceptable information that lets us proceed a bit further.

Lemma 3.2. Let $D \subset \mathbb{R}^{n}$ be a bounded region with a $C^{2}$-boundary $\partial D$. Then

$$
|\hat{n}(x) \cdot(x-y)|<L|x-y|^{2} \quad \text { for all } x, y \in \partial D,
$$

where $L$ is some positive constant.

Proof. For proof consult [4].

Corollary 3.3. The kernel $h$ (and $h_{0}$ ) belongs to space $L^{2}\left(\partial \Omega_{n} \times \partial \Omega_{n}\right)$.

Proof. Using Lemma 3.2 one easily sees that

$$
\begin{aligned}
|h(x, y)| & \leqslant\left|h_{0}(x, y)\right| \\
& \leqslant \frac{C_{n}}{|x-y|^{n-3}},
\end{aligned}
$$

which clearly proves the statement for $n=2$ and $n=3$.

It is a straightforward consequence of Corollary 3.3 and the Schwarz inequality that the operator $\mathcal{G}$ belongs to $\mathcal{L}\left(L^{2}\left(\partial \Omega_{n}\right)\right)$. This kind of weak result is not, however, by itself satisfactory for our purposes and so we state something stronger, and omit the proof.

Theorem 3.4. $\mathcal{G}: L^{2}\left(\partial \Omega_{n}\right) \rightarrow L^{2}\left(\partial \Omega_{n}\right)$ is a compact linear operator for which

$$
\|\mathcal{G}\|_{\mathcal{L}\left(L^{2}\left(\partial \Omega_{n}\right)\right)} \leqslant\|h\|_{L^{2}\left(\partial \Omega_{n}\right) \times L^{2}\left(\partial \Omega_{n}\right)} .
$$

Proof. See $[\mathbf{1 1}]$.

Next we investigate the kernel $h_{0}$ in more detail.

Lemma 3.5. For kernel $h_{0}$ it is true that

$$
\int_{\partial \Omega_{n}} h_{0}(x, y) \mathrm{d} S(x)=\int_{\partial \Omega_{n}} h_{0}(x, y) \mathrm{d} S(y)=-1 .
$$


Proof. The first equality is apparent because of the symmetry of $h_{0}$. The second equality is easily proved by proceeding in the opposite direction to the one we followed in $\S 2$ : we use the relation between elements $\mathrm{d} S(y)$ and $\mathrm{d} s(\hat{\theta})$, namely

$$
\hat{\theta} \cdot \hat{n}(y) \mathrm{d} S(y)=|x-y|^{n-1} \mathrm{~d} s(\hat{\theta}), \quad \hat{\theta}=\frac{x-y}{|x-y|},
$$

change the integration to be over directions rather than the boundary $\partial \Omega_{n}$ and we are already there:

$$
\begin{aligned}
\int_{\partial \Omega_{n}} h_{0}(x, y) \mathrm{d} S(y) & =\frac{n-1}{\left|S^{n-2}\right|} \int_{\partial \Omega_{n}} v(x, y) \frac{(\hat{n}(x) \cdot(x-y))(\hat{n}(y) \cdot(x-y))}{|x-y|^{n+1}} \mathrm{~d} S(y) \\
& =\frac{n-1}{\left|S^{n-2}\right|} \int_{\{\hat{\theta} \cdot \hat{n}(x)<0\}} \hat{n}(x) \cdot \hat{\theta} \mathrm{d} s(\hat{\theta}) \\
& =-1 .
\end{aligned}
$$

One should note that the above result tells us the following: if the non-scattering region $\Omega_{n}$ is also non-absorbing, meaning that $\tilde{\mu}_{\mathrm{a}}=0$, a unit input flux of direct current $(k=0)$ on the boundary $\partial \Omega_{n}$ should, according to our model, result in a unit output flux of direct current on $\partial \Omega_{n}$.

Despite the interesting note we made above, we will assume from now on that $\tilde{\mu}_{\mathrm{a}}>0$. From the physical point of view, this kind of assumption sounds reasonable, and from the mathematical point of view, it keeps us out of trouble when proving the existence of the inverse operators. We end the discussion on the basic properties of the operator $\mathcal{G}$ with a neat norm estimate.

Lemma 3.6. The operator $\mathcal{G}$ belongs to space $\mathcal{L}\left(L^{1}\left(\partial \Omega_{n}\right)\right)$, with the norm estimate

$$
\|\mathcal{G}\|_{\mathcal{L}\left(L^{1}\left(\partial \Omega_{n}\right)\right)}<1 .
$$

Proof. Let $u \in L^{1}\left(\partial \Omega_{n}\right)$. Then it holds that

$$
\begin{aligned}
\|\mathcal{G} u\|_{L^{1}\left(\partial \Omega_{n}\right)} & =\int_{\partial \Omega_{n}}\left|\int_{\partial \Omega_{n}} h(x, y) u(y) \mathrm{d} S(y)\right| \mathrm{d} S(x) \\
& <\int_{\partial \Omega_{n}} \int_{\partial \Omega_{n}}\left|h_{0}(x, y)\right||u(y)| \mathrm{d} S(y) \mathrm{d} S(x) \\
& =\int_{\partial \Omega_{n}}|u(y)| \int_{\partial \Omega_{n}}\left|h_{0}(x, y)\right| \mathrm{d} S(x) \mathrm{d} S(y) \\
& =\|u\|_{L^{1}\left(\partial \Omega_{n}\right)},
\end{aligned}
$$

where we used Fubini's Theorem, Lemma 3.5 and the fact that $h_{0} \leqslant 0$ (draw a picture). 
Note that the above result actually proves that the inverse operators $(I \pm \mathcal{G})^{-1} \in$ $L^{1}\left(\partial \Omega_{n}\right)$ exist. Together with the Fredholm alternative, Lemma 3.6 could also be used to prove the existence of the operators $(I \pm \mathcal{G})^{-1} \in L^{2}\left(\partial \Omega_{n}\right)$. However, since we obtain a result that is a little bit stronger as a byproduct of spectral analysis in the next subsection, we postpone the more profound considerations of this matter.

\subsection{Some spectral analysis}

Since $\mathcal{G}: L^{2}\left(\partial \Omega_{n}\right) \rightarrow L^{2}\left(\partial \Omega_{n}\right)$ is compact, spectral analysis provides an efficient way of gathering information on its behaviour. The following easy variant of the well-known theorem concerning the canonical form of compact operators [10] forms the basis of our work.

Theorem 3.7. Let $\mathcal{H}$ be a Hilbert space and let $\mathcal{T}: \mathcal{H} \rightarrow \mathcal{H}$ be linear and compact. Assume that $\mathcal{N}(\mathcal{T})$ and $\mathcal{N}\left(\mathcal{T}^{*}\right)$ are isomorphic. Then there exists orthonormal bases $\left\{\phi_{k}\right\}$ and $\left\{\psi_{k}\right\}$ of $\mathcal{H}$ consisting of the eigenfunctions of $\mathcal{T}^{*} \mathcal{T}$ and $\mathcal{T} \mathcal{T}^{*}$, respectively, with the properties

$$
\mathcal{T} \phi_{k}=\lambda_{k} \psi_{k}, \quad \mathcal{T}^{*} \psi_{k}=\lambda_{k} \phi_{k}, \quad \text { for all } k \in \mathbb{N} .
$$

Further, if $\mu_{k} \in \mathbb{R}^{+}$is the eigenvalue of $\mathcal{T}^{*} \mathcal{T}$ corresponding to $\phi_{k}$, then $\lambda_{k}=\mu_{k}^{1 / 2}$.

The next lemma translates Theorem 3.7 into a more suitable form.

Lemma 3.8. Let $\mathcal{H}$ be a Hilbert space and let $\mathcal{T}: \mathcal{H} \rightarrow \mathcal{H}$ be a compact linear operator for which

$$
\mathcal{T}^{*} \phi=\overline{\mathcal{T} \bar{\phi}} \text { for all } \phi \in \mathcal{H}
$$

For $\mathcal{T}$ there exists an orthonormal basis $\left\{\phi_{k}\right\}$ of $\mathcal{H}$ such that

$$
\mathcal{T} \phi_{k}=\lambda_{k} \bar{\phi}_{k}, \quad \mathcal{T}^{*} \bar{\phi}_{k}=\bar{\lambda}_{k} \phi_{k}, \quad \text { for all } k \in \mathbb{N},
$$

where $\left|\lambda_{k}\right|=\mu^{1 / 2}$ for some eigenvalue $\mu$ of $\mathcal{T}^{*} \mathcal{T}$.

Proof. First of all, note that in this case an isomorphism between $\mathcal{N}(\mathcal{T})$ and $\mathcal{N}\left(\mathcal{T}^{*}\right)$ is provided by complex conjugation.

Let $\left\{\phi_{k}\right\}$ and $\left\{\lambda_{k}^{2}\right\}$ be sets of orthonormal eigenfunctions and eigenvalues corresponding to the compact self-adjoint operator $\mathcal{T}^{*} \mathcal{T}$, respectively. Thus, we may write

$$
\mathcal{T}^{*} \mathcal{T} \phi_{k}=\lambda_{k}^{2} \phi_{k}
$$

Taking the complex conjugate of both sides and keeping (3.3) in mind we get

$$
\mathcal{T} \mathcal{T}^{*} \bar{\phi}_{k}=\lambda_{k}^{2} \bar{\phi}_{k}
$$

and so it is clear that $\left\{\bar{\phi}_{k}\right\}$ is an orthonormal set of the eigenfunctions of $\mathcal{T T}^{*}$, forming a basis of $\mathcal{H}$, with the corresponding eigenvalues $\left\{\lambda_{k}^{2}\right\}$.

It is easy to see that the eigenfunctions of $\mathcal{T}^{*} \mathcal{T}$ corresponding to the zero eigenvalue satisfy equations (3.4). Hence, let $\phi_{k_{1}}, \ldots, \phi_{k_{N}} \in\left\{\phi_{k}\right\}$ be the eigenfunctions corresponding to the eigenvalue $\lambda_{k}^{2}>0$. From what was stated above and Theorem 3.7 it 
is quite clear that $\mathcal{T}$ maps the subspace $\mathcal{M}=\operatorname{span}\left\{\phi_{k_{1}}, \ldots, \phi_{k_{N}}\right\}$ onto the subspace $\overline{\mathcal{M}}=\operatorname{span}\left\{\bar{\phi}_{k_{1}}, \ldots, \bar{\phi}_{k_{N}}\right\}$. Because $\mathcal{M}$ and $\overline{\mathcal{M}}$ are finite dimensional, we know that the restriction of $\mathcal{T}$ to $\mathcal{M}$, namely $\left.\mathcal{T}\right|_{\mathcal{M}}$, may be represented in matrix form with respect to the bases $\left\{\phi_{k_{1}}, \ldots, \phi_{k_{N}}\right\}$ and $\left\{\bar{\phi}_{k_{1}}, \ldots, \bar{\phi}_{k_{N}}\right\}$. Hence, we can write

$$
\left.\mathcal{T}\right|_{\mathcal{M}} \phi_{k_{l}}=\lambda_{k} \sum_{j=1}^{N} t_{j l} \bar{\phi}_{k_{j}}, \quad l=1, \ldots, N,
$$

where $T=\left(t_{j l}\right)$ is the matrix corresponding to $\left.\mathcal{T}\right|_{\mathcal{M}}$. Taking complex conjugates and keeping (3.3) in mind, we get

$$
\left.\mathcal{T}^{*}\right|_{\mathcal{M}} \bar{\phi}_{k_{l}}=\lambda_{k} \sum_{j=1}^{N} \bar{t}_{j l} \phi_{k_{j}}, \quad l=1, \ldots, N .
$$

Thus, $\left.\mathcal{T}^{*}\right|_{\overline{\mathcal{M}}}$ has the matrix representation $\bar{T}=\left(\bar{t}_{j l}\right)$. Now it follows easily from equation (3.3) that $T$ is symmetric, and so equation (3.5) tells us that

$$
\frac{1}{\lambda_{k}^{2}} T^{H} T=\frac{1}{\lambda_{k}^{2}} \bar{T} T=I,
$$

which means that $\left(1 / \lambda_{k}\right) T$ is unitary and therefore has orthonormal eigenvectors $\left\{v_{1}, \ldots\right.$, $\left.v_{N}\right\}$ that span $\mathbb{C}^{N}$. Since $\left(1 / \lambda_{k}\right) T$ is unitary, the corresponding eigenvalues $\left\{\nu_{1}, \ldots, \nu_{N}\right\}$ are, naturally, of magnitude one.

Now we form a new set of functions, namely $\left\{\tilde{\phi}_{k_{1}}, \ldots, \tilde{\phi}_{k_{N}}\right\}$, by

$$
\tilde{\phi}_{k_{l}}=\left[\phi_{k_{1}}, \ldots, \phi_{k_{N}}\right] v_{l}, \quad l=1, \ldots N .
$$

One can easily see that these new functions are still orthonormal because vectors $\left\{v_{1}, \ldots\right.$, $\left.v_{N}\right\}$ are. In addition, it holds that

$$
\mathcal{T} \tilde{\phi}_{k_{l}}=\lambda_{k_{l}} \overline{\tilde{\phi}}_{k_{l}}
$$

where $\lambda_{k_{l}}=\nu_{l} \lambda_{k}$, since $\left\{v_{1}, \ldots, v_{N}\right\}$ are eigenvectors of $T$.

Finally, if we repeat the same procedure for all $\lambda_{k}$ and combine the new eigenfunctions and eigenvalues, we have arrived with an orthonormal basis of $\mathcal{H}$ that has just the qualities we were looking for.

Note that since $\mathcal{G}$ is a compact integral operator with a symmetric kernel, it satisfies the assumptions of the previous lemma, which will be used in the next subsection when we state and prove an important positiveness result. Next we will, however, finish the postponed analysis of the existence of $(I \pm \mathcal{G})^{-1} \in L^{2}\left(\partial \Omega_{n}\right)$.

Lemma 3.9. The eigenvalues of the positive self-adjoint operator $\mathcal{G}^{*} \mathcal{G} \in \mathcal{L}\left(L^{2}\left(\partial \Omega_{n}\right)\right)$ lie in the closed interval $\left[0, \rho^{2}\right]$, where $\rho=\|\mathcal{G}\|_{\mathcal{L}\left(L^{1}\left(\partial \Omega_{n}\right)\right)}<1$. 
Proof. Let $\mu \in \mathbb{R}^{+}$be an eigenvalue of $\mathcal{G}^{*} \mathcal{G}$ and let $\phi \in L^{2}\left(\partial \Omega_{n}\right)$ be the corresponding eigenfunction. Then, from the definition of the norm, we get

$$
\begin{aligned}
\mu\|\phi\|_{L^{1}\left(\partial \Omega_{n}\right)} & =\left\|\mathcal{G}^{*} \mathcal{G} \phi\right\|_{L^{1}\left(\partial \Omega_{n}\right)} \\
& \leqslant\|\mathcal{G}\|_{\mathcal{L}\left(L^{1}\left(\partial \Omega_{n}\right)\right)}^{2}\|\phi\|_{L^{1}\left(\partial \Omega_{n}\right)}<\infty
\end{aligned}
$$

since $L^{2}\left(\partial \Omega_{n}\right) \subset L^{1}\left(\partial \Omega_{n}\right)$. Thus

$$
\mu \leqslant\|\mathcal{G}\|_{\mathcal{L}\left(L^{1}\left(\partial \Omega_{n}\right)\right)}^{2}<1,
$$

according to Lemma 3.6.

Theorem 3.10. The operators $I \pm \mathcal{G}: L^{2}\left(\partial \Omega_{n}\right) \rightarrow L^{2}\left(\partial \Omega_{n}\right)$ have bounded inverses $(I \pm \mathcal{G})^{-1} \in \mathcal{L}\left(L^{2}\left(\partial \Omega_{n}\right)\right)$. Further, it holds that

$$
(I \pm \mathcal{G})^{-1}=\sum_{k=0}^{\infty}(\mp \mathcal{G})^{k} .
$$

Proof. Let $\left\{\mu_{k}\right\}$ be the eigenvalues of $\mathcal{G}^{*} \mathcal{G}$. It is an easy consequence of Theorem 3.7 that

$$
\|\mathcal{G}\|_{\mathcal{L}\left(L^{2}\left(\partial \Omega_{n}\right)\right)} \leqslant \sup _{k \in \mathbb{N}} \sqrt{\mu_{k}} \leqslant \rho
$$

where $\rho<1$ is the constant introduced in Lemma 3.9. Now the claim follows from elementary functional analysis $[\mathbf{9}]$.

It is interesting to notice that the standard norm estimate (3.1) is, actually, much worse than (3.6).

\subsection{A positiveness result}

Finally, we are about to prove the main result of this section. In the next section, this result will be a real aid when we set out to prove the existence of the weak solution of the problem introduced in Definition 2.6. First, however, we need a little lemma.

Lemma 3.11. For $\mathcal{G}$ let $\left\{\phi_{k}\right\}$ be the orthonormal set introduced in Lemma 3.8 and let $\left\{\lambda_{k}\right\}$ be the corresponding set of singular values. Then the set $\left\{\phi_{k}-\lambda_{k} \bar{\phi}_{k}\right\}$ forms a basis of $L^{2}\left(\partial \Omega_{n}\right)$.

Proof. Let $u \in L^{2}\left(\partial \Omega_{n}\right)$. Since $\left\{\phi_{k}\right\}$ forms an orthonormal basis for $L^{2}\left(\partial \Omega_{n}\right)$ and by Theorem $3.10(I-\mathcal{G})^{-1}$ exists, we may write

$$
(I-\mathcal{G})^{-1} u=\sum_{k=1}^{\infty}\left((I-\mathcal{G})^{-1} u, \phi_{k}\right) \phi_{k} .
$$

Thus, by operating with $I-\mathcal{G}$, we get

$$
u=\sum_{k=1}^{\infty}\left((I-\mathcal{G})^{-1} u, \phi_{k}\right)\left(\phi_{k}-\lambda_{k} \bar{\phi}_{k}\right),
$$

where we used Lemma 3.8.

Furthermore, $\left\{\phi_{k}-\lambda_{k} \bar{\phi}_{k}\right\}$ is a linearly independent set, since $I-\mathcal{G}$ is injective. 
Theorem 3.12. The operator $(I-\mathcal{G})^{-1}(I+\mathcal{G})$ is positive definite in the real part, meaning that for any $u \in L^{2}\left(\partial \Omega_{n}\right), u \neq 0$,

$$
\operatorname{Re}\left((I-\mathcal{G})^{-1}(I+\mathcal{G}) u, u\right)>0
$$

Proof. Again, let $\left\{\phi_{k}\right\}$ be the orthonormal set introduced in Lemma 3.8 and let $\left\{\lambda_{k}\right\}$ be the corresponding set of singular values. With trivial algebraic manipulation, one sees that

$$
(I+\mathcal{G})\left(\phi_{k}-\lambda_{k} \bar{\phi}_{k}\right)=\phi_{k}-\lambda_{k} \mathcal{G} \bar{\phi}_{k}=(I-\mathcal{G})\left(\phi_{k}+\lambda_{k} \bar{\phi}_{k}\right)
$$

Hence,

$$
(I-\mathcal{G})^{-1}(I+\mathcal{G})\left(\phi_{k}-\lambda_{k} \bar{\phi}_{k}\right)=\left(\phi_{k}+\lambda_{k} \bar{\phi}_{k}\right)
$$

Let $u \in L^{2}\left(\partial \Omega_{n}\right)$. Since $\left\{\phi_{k}-\lambda_{k} \bar{\phi}_{k}\right\}$ forms a basis of $L^{2}\left(\partial \Omega_{n}\right), u$ may be presented in the form

$$
u=\sum_{k=1}^{\infty} c_{k}\left(\phi_{k}-\lambda_{k} \bar{\phi}_{k}\right)
$$

where, according to the proof of Lemma 3.12, $c_{k}=\left((I-\mathcal{G})^{-1} u, \phi_{k}\right)$. With the help of (3.7), we can now write

$$
\begin{aligned}
\left((I-\mathcal{G})^{-1}(I+\mathcal{G}) u, u\right)= & \left(\sum_{k=1}^{\infty} c_{k}\left(\phi_{k}+\lambda_{k} \bar{\phi}_{k}\right), \sum_{j=1}^{\infty} c_{j}\left(\phi_{j}-\lambda_{j} \bar{\phi}_{j}\right)\right) \\
= & \sum_{k=1}^{\infty}\left(c_{k}\left(\phi_{k}+\lambda_{k} \bar{\phi}_{k}\right), c_{k}\left(\phi_{k}-\lambda_{k} \bar{\phi}_{k}\right)\right) \\
& +\sum_{k=1}^{\infty} \sum_{j \neq k}\left(c_{k}\left(\phi_{k}+\lambda_{k} \bar{\phi}_{k}\right), c_{j}\left(\phi_{j}-\lambda_{j} \bar{\phi}_{j}\right)\right) .
\end{aligned}
$$

To start with, let us have a closer look at the first sum in (3.8):

$$
\begin{aligned}
\sum_{k=1}^{\infty}\left(c_{k}\left(\phi_{k}+\lambda_{k} \bar{\phi}_{k}\right),\right. & \left.c_{k}\left(\phi_{k}-\lambda_{k} \bar{\phi}_{k}\right)\right) \\
& =\sum_{k=1}^{\infty}\left|c_{k}\right|^{2}\left(\left\|\phi_{k}\right\|^{2}-\left|\lambda_{k}\right|^{2}\left\|\bar{\phi}_{k}\right\|^{2}+\lambda_{k}\left(\bar{\phi}_{k}, \phi_{k}\right)-\bar{\lambda}_{k}\left(\phi_{k}, \bar{\phi}_{k}\right)\right) \\
& =\sum_{k=1}^{\infty}\left|c_{k}\right|^{2}\left(1-\left|\lambda_{k}\right|^{2}\right)+2 \mathrm{i} \sum_{k=1}^{\infty}\left|c_{k}\right|^{2} \operatorname{Im}\left(\lambda_{k}\left(\bar{\phi}_{k}, \phi_{k}\right)\right)
\end{aligned}
$$


Next we shall attack the second term of (3.8):

$$
\begin{aligned}
\sum_{k=1}^{\infty} \sum_{j \neq k}\left(c_{k}\left(\phi_{k}+\lambda_{k} \bar{\phi}_{k}\right), c_{j}\left(\phi_{j}-\lambda_{j} \bar{\phi}_{j}\right)\right) & =\sum_{k=1}^{\infty} \sum_{j \neq k} c_{k} \bar{c}_{j}\left(\lambda_{k}\left(\bar{\phi}_{k}, \phi_{j}\right)-\bar{\lambda}_{j}\left(\phi_{k}, \bar{\phi}_{j}\right)\right) \\
& =\sum_{k=1}^{\infty} \sum_{j>k}\left(c_{k} \bar{c}_{j}\left(\lambda_{k}\left(\bar{\phi}_{k}, \phi_{j}\right)-\bar{\lambda}_{j}\left(\phi_{k}, \bar{\phi}_{j}\right)\right)\right. \\
& \left.+c_{j} \bar{c}_{k}\left(\lambda_{j}\left(\bar{\phi}_{j}, \phi_{k}\right)-\bar{\lambda}_{k}\left(\phi_{j}, \bar{\phi}_{k}\right)\right)\right) \\
& =2 \mathrm{i} \sum_{k=1}^{\infty} \sum_{j>k} \operatorname{Im}\left(c_{k} \bar{c}_{j}\left(\lambda_{k}\left(\bar{\phi}_{k}, \phi_{j}\right)-\bar{\lambda}_{j}\left(\phi_{k}, \bar{\phi}_{j}\right)\right)\right) .
\end{aligned}
$$

Combining (3.9) and (3.10), we obtain

$$
\left((I-\mathcal{G})^{-1}(I+\mathcal{G}) u, u\right)=\sum_{k=1}^{\infty}\left|c_{k}\right|^{2}\left(1-\left|\lambda_{k}\right|^{2}\right)+\mathrm{i} C,
$$

where $C$ is a real constant. Finally, Lemmas 3.8 and 3.9 tell us that $1-\left|\lambda_{k}\right|^{2}>0$ for every $k>0$, which concludes the proof.

Note that the proof of Lemma 3.12 can be presented without the information provided by Lemma 3.8. However, Lemma 3.8 is interesting by itself and it provides useful insight into the actual shape of operator $\mathcal{G}$.

\section{The weak problem}

In this section we will formulate the weak direct problem corresponding to the classical one, introduced in Definition 2.6, and prove that there exists a unique solution for it.

If $\varphi$ satisfies equation (2.18) in $\Omega \backslash \bar{\Omega}_{n}$, it also satisfies

$$
\int_{\Omega \backslash \bar{\Omega}_{n}} \nabla \cdot K \nabla \varphi \bar{v} \mathrm{~d} x+\int_{\Omega \backslash \bar{\Omega}_{n}}\left(\mathrm{i} k-\mu_{\mathrm{a}}\right) \varphi \bar{v} \mathrm{~d} x=0
$$

for every smooth $v$. Using the product rule, we can write this as

$$
\int_{\Omega \backslash \bar{\Omega}_{n}} \nabla \cdot(K \nabla \varphi \bar{v}) \mathrm{d} x-\int_{\Omega \backslash \bar{\Omega}_{n}} K \nabla \varphi \cdot \nabla \bar{v} \mathrm{~d} x+\int_{\Omega \backslash \bar{\Omega}_{n}}\left(\mathrm{i} k-\mu_{\mathrm{a}}\right) \varphi \bar{v} \mathrm{~d} x=0,
$$

which, on the other hand, is equivalent to

$$
\begin{aligned}
\int_{\partial \Omega} \hat{n} \cdot(K \nabla \varphi \bar{v}) \mathrm{d} S+\int_{\partial \Omega_{n}} \hat{n} \cdot(K \nabla \varphi \bar{v}) \mathrm{d} S-\int_{\Omega \backslash \bar{\Omega}_{n}} K \nabla \varphi \cdot \nabla \bar{v} \mathrm{~d} x \\
\quad+\int_{\Omega \backslash \bar{\Omega}_{n}}\left(\mathrm{i} k-\mu_{\mathrm{a}}\right) \varphi \bar{v} \mathrm{~d} x=0
\end{aligned}
$$


due to the divergence theorem of Gauss. Note that $\hat{n}$ is the unit normal vector pointing out of $\Omega \backslash \bar{\Omega}_{n}$. Next we use the boundary conditions (2.19) and (2.20), change our matrix notation, and rewrite (4.1) as

$$
\begin{aligned}
\int_{\Omega \backslash \bar{\Omega}_{n}} \nabla \varphi^{\mathrm{T}} K^{\mathrm{T}} \nabla \bar{v} \mathrm{~d} x & +\int_{\Omega \backslash \bar{\Omega}_{n}}\left(\mu_{\mathrm{a}}-\mathrm{i} k\right) \varphi \bar{v} \mathrm{~d} x+2 \gamma \int_{\partial \Omega} \varphi \bar{v} \mathrm{~d} S \\
& +2 \gamma \int_{\partial \Omega_{n}}(I-\mathcal{G})^{-1}(I+\mathcal{G}) \varphi \bar{v} \mathrm{~d} S=-2 \int_{\partial \Omega} \Phi_{\text {in }} \bar{v} \mathrm{~d} S .
\end{aligned}
$$

As we can see, equation (4.2) makes sense even when both $\varphi$ and $v$ have only one weak derivative that belongs to $L^{2}\left(\Omega \backslash \bar{\Omega}_{n}\right)$. The input flux coming from some outer source, $\Phi_{\text {in }}$, may be even less smooth - it seems to be enough that $\Phi_{\text {in }} \in L^{2}(\partial \Omega)$. These observations make the following definition reasonable.

Definition 4.1. Let $\Phi_{\text {in }}$ belong to $L^{2}(\partial \Omega)$ and suppose that $0<c_{\mathrm{a}}<\mu_{\mathrm{a}}<C_{\mathrm{a}}$ and $0<\mu_{\mathrm{s}}<C_{\mathrm{s}}$ are measurable real functions. The direct weak problem in the strongly scattering region $\Omega \backslash \bar{\Omega}_{n}$ is to find the function $\varphi \in H^{1}\left(\Omega \backslash \bar{\Omega}_{n}\right)$ satisfying equation (4.2) for every $v \in H^{1}\left(\Omega \backslash \bar{\Omega}_{n}\right)$.

\subsection{An existence and uniqueness result}

To make Definition 4.1 permissible, we will have to prove that there really exists a unique solution $\varphi \in H^{1}\left(\Omega \backslash \bar{\Omega}_{n}\right)$ to equation (4.2). We start our battle by rewriting (4.2) as

$$
B(\varphi, v):=B_{1}(\varphi, v)+B_{2}(\varphi, v)+B_{3}(\varphi, v)+B_{4}(\varphi, v)=f(v), \quad v \in H^{1}\left(\Omega \backslash \bar{\Omega}_{n}\right),
$$

where $B_{1}$ corresponds to the first term in (4.2) and so on.

Our plan is to prove the unique existence of the solution by means of the well-known Lax-Milgram Lemma [13]. Hence, our task is to prove that the sesquilinear functional $B$ is bounded and coercive, and that the functional $f$ is bounded. We will start with a little lemma concerning the matrix $K$ and after that tackle the boundedness of $B$.

Lemma 4.2. The matrix $K(x) \in \mathbb{C}^{n \times n}$ defined in (2.12) is symmetric and has real orthonormal eigenvectors $\left\{w_{1}(x), \ldots, w_{n}(x)\right\}$, for which the corresponding eigenvalues $\left\{\lambda_{1}(x), \ldots, \lambda_{n}(x)\right\}$ are strictly positive in the real part, meaning in this case that $\operatorname{Re} \lambda_{j} \geqslant$ $c_{\lambda}>0$ for all $j=1, \ldots, n$. Furthermore, $|K(x)| \leqslant\left(1 / n c_{\mathrm{a}}\right)$ for all $x \in \Omega \backslash \bar{\Omega}_{n}$.

Proof. According to Lemma 2.4, the symmetric matrix $I-B(x) \in \mathbb{R}^{n \times n}$ has positive eigenvalues $0 \leqslant \lambda_{j}^{0}(x) \leqslant 2, j=1, \ldots, n$, for which the corresponding eigenvectors $\left\{w_{1}(x), \ldots, w_{n}(x)\right\} \subset \mathbb{R}^{n}$ may be chosen orthonormal. Thus, the matrix

$$
K^{-1}(x)=n\left(\left(\mu_{\mathrm{a}}(x)-\mathrm{i} k\right) I+(I-B(x)) \mu_{\mathrm{s}}(x)\right)
$$

has the same eigenvectors as $I-B(x)$ with the corresponding eigenvalues

$$
n\left(\mu_{\mathrm{s}}(x) \lambda_{j}^{0}(x)+\mu_{\mathrm{a}}(x)-\mathrm{i} k\right), \quad j=1, \ldots, n .
$$


In addition, $K^{-1}$ is symmetric. Hence, $K(x)$ is symmetric, and the orthonormal vectors $\left\{w_{1}(x), \ldots, w_{n}(x)\right\}$ are also eigenvectors of $K(x)$ with the corresponding eigenvalues

$$
\begin{aligned}
\lambda_{j}(x) & :=\xi_{j}(x)+\mathrm{i} \eta_{j}(x) \\
& =\frac{1}{n\left(\left(\mu_{\mathrm{s}}(x) \lambda_{j}^{0}(x)+\mu_{\mathrm{a}}(x)\right)^{2}+k^{2}\right)}\left(\mu_{\mathrm{s}}(x) \lambda_{j}^{0}(x)+\mu_{\mathrm{a}}(x)+\mathrm{i} k\right) .
\end{aligned}
$$

Since $0<\mu_{\mathrm{s}}<C_{\mathrm{s}}, c_{\mathrm{a}}<\mu_{\mathrm{a}}<C_{\mathrm{a}}$ and $0 \leqslant \lambda_{j}^{0}(x) \leqslant 2$, the positiveness of the real part follows easily. On the other hand, equation (4.4) tells us that for every $j=1, \ldots, n$,

$$
\begin{aligned}
\left|\lambda_{j}(x)\right| & \leqslant \frac{1}{n \mu_{\mathrm{a}}(x)} \\
& \leqslant \frac{1}{n c_{\mathrm{a}}},
\end{aligned}
$$

from which the result follows, since $|K(x)|=\max _{j}\left\{\left|\lambda_{j}(x)\right|\right\}$.

Lemma 4.3. The sesquilinear form $B: H^{1}\left(\Omega \backslash \bar{\Omega}_{n}\right) \times H^{1}\left(\Omega \backslash \bar{\Omega}_{n}\right) \rightarrow \mathbb{C}$, defined by equations (4.2) and (4.3), is bounded.

Proof. Let $u, v \in H^{1}\left(\Omega \backslash \bar{\Omega}_{n}\right)$. First, we tackle $B_{1}$ :

$$
\begin{aligned}
\left|B_{1}(u, v)\right| & \leqslant \sup _{x \in \Omega \backslash \bar{\Omega}_{n}}\{|K(x)|\}\left(\int_{\Omega \backslash \bar{\Omega}_{n}}|\nabla u|^{2} \mathrm{~d} x\right)^{1 / 2}\left(\int_{\Omega \backslash \bar{\Omega}_{n}}|\nabla v|^{2} \mathrm{~d} x\right)^{1 / 2} \\
& \leqslant \frac{1}{n c_{\mathrm{a}}}\|u\|_{H^{1}\left(\Omega \backslash \bar{\Omega}_{n}\right)}\|v\|_{H^{1}\left(\Omega \backslash \bar{\Omega}_{n}\right)},
\end{aligned}
$$

where we used the Schwarz inequality and Lemma 4.2. Next, we use the same weaponry on $B_{2}$ :

$$
\begin{aligned}
\left|B_{2}(u, v)\right| & \leqslant \sup _{\Omega \backslash \bar{\Omega}_{n}}\left\{\left|\mu_{\mathrm{a}}(x)-\mathrm{i} k\right|\right\}\left(\int_{\Omega \backslash \bar{\Omega}_{n}}|u|^{2} \mathrm{~d} x\right)^{1 / 2}\left(\int_{\Omega \backslash \bar{\Omega}_{n}}|v|^{2} \mathrm{~d} x\right)^{1 / 2} \\
& \leqslant\left(C_{\mathrm{a}}^{2}+k^{2}\right)^{1 / 2}\|u\|_{H^{1}\left(\Omega \backslash \bar{\Omega}_{n}\right)}\|v\|_{H^{1}\left(\Omega \backslash \bar{\Omega}_{n}\right)} .
\end{aligned}
$$

Then there is $B_{3}$, which we can attack with the help of the trace theorem [6] and, of course, the Schwarz inequality:

$$
\begin{aligned}
\left|B_{3}(u, v)\right| & \leqslant 2 \gamma\left(\int_{\partial \Omega}|u|^{2} \mathrm{~d} x\right)^{1 / 2}\left(\int_{\partial \Omega}|v|^{2} \mathrm{~d} x\right)^{1 / 2} \\
& \leqslant C\|u\|_{H^{1}\left(\Omega \backslash \bar{\Omega}_{n}\right)}\|v\|_{H^{1}\left(\Omega \backslash \bar{\Omega}_{n}\right)} .
\end{aligned}
$$

Using the Schwarz inequality, the boundedness of the operators $I+\mathcal{G}$ and $(I-\mathcal{G})^{-1}$ and the trace theorem, it follows easily for $B_{4}$ that

$$
\begin{aligned}
\left|B_{4}(u, v)\right| & \leqslant 2 \gamma\left\|(I-\mathcal{G})^{-1}\right\|_{\mathcal{L}\left(L^{2}\left(\partial \Omega_{n}\right)\right)}\|I+\mathcal{G}\|_{\mathcal{L}\left(L^{2}\left(\partial \Omega_{n}\right)\right)}\|u\|_{L^{2}\left(\partial \Omega_{n}\right)}\|v\|_{L^{2}\left(\partial \Omega_{n}\right)} \\
& \leqslant C\|u\|_{H^{1}\left(\Omega \backslash \bar{\Omega}_{n}\right)}\|v\|_{H^{1}\left(\Omega \backslash \bar{\Omega}_{n}\right)} .
\end{aligned}
$$

Finally, the boundedness of the whole functional $B$ follows from the triangle inequality. 
Next, we will prove the coercivity property of $B$.

Lemma 4.4. The sesquilinear form $B: H^{1}\left(\Omega \backslash \bar{\Omega}_{n}\right) \times H^{1}\left(\Omega \backslash \bar{\Omega}_{n}\right) \rightarrow \mathbb{C}$, defined by equations (4.2) and (4.3), is coercive.

Proof. Let $u \in H^{1}\left(\Omega \backslash \bar{\Omega}_{n}\right)$. First of all, notice that

$$
|B(u, u)| \geqslant \operatorname{Re}\left(B_{1}(u, u)+B_{2}(u, u)+B_{3}(u, u)+B_{4}(u, u)\right) .
$$

We begin by proving that the real part of $B_{1}(u, u)+B_{2}(u, u)$ is coercive by itself. Notice that for almost every fixed $x \in \Omega \backslash \bar{\Omega}_{n}$ we can give $\nabla u(x)$ as a linear combination of the eigenvectors of $K(x)$ introduced in Lemma 4.2:

$$
\nabla u(x)=\sum_{j=1}^{n} c_{j}(x) w_{j}(x) .
$$

Thus, for a fixed $x$ it holds almost everywhere that

$$
\begin{aligned}
\nabla u^{\mathrm{T}}(x) K(x) \nabla \bar{u}(x) & =\sum_{j=1}^{n} c_{j}(x) w(x)_{j}^{\mathrm{T}} \sum_{k=1}^{n} \lambda_{k}(x) \bar{c}_{k}(x) w_{k}(x) \\
& =\sum_{j=1}^{n} \lambda_{j}(x)\left|c_{j}(x)\right|^{2}
\end{aligned}
$$

where we used the orthonormality of $w_{j}(x), j=1, \ldots, n$. Hence, almost everywhere

$$
\begin{aligned}
\operatorname{Re} \nabla u^{\mathrm{T}}(x) K(x) \nabla u(x) & =\sum_{j=1}^{n} \xi_{j}(x)\left|c_{j}(x)\right|^{2} \\
& \geqslant \min _{1 \leqslant l \leqslant n} \xi_{l}(x) \sum_{j=1}^{n}\left|c_{j}(x)\right|^{2} \\
& =\min _{1 \leqslant l \leqslant n} \xi_{l}(x)|\nabla u(x)|^{2},
\end{aligned}
$$

where we used the notation of Lemma 4.2. By using the symmetry of $K$ and Lemma 4.2, it follows that

$$
\begin{aligned}
\operatorname{Re} B_{1}(u, u) & \geqslant \inf _{\{1, \ldots, n\} \times \Omega \backslash \bar{\Omega}_{n}}\left\{\xi_{l}(x)\right\} \int_{\Omega \backslash \bar{\Omega}_{n}}|\nabla u|^{2} \mathrm{~d} x \\
& \geqslant c_{\lambda}\|\mid \nabla u\|_{L^{2}\left(\Omega \backslash \bar{\Omega}_{n}\right)}^{2} .
\end{aligned}
$$

On the other hand, we can estimate $B_{2}$ in the following way:

$$
\begin{aligned}
\operatorname{Re} B_{2}(u, u) & \geqslant \inf _{x \in \Omega \backslash \bar{\Omega}_{n}}\left\{\mu_{\mathrm{a}}(x)\right\} \int_{\Omega \backslash \bar{\Omega}_{n}}|u|^{2} \mathrm{~d} x \\
& \geqslant c_{\mathrm{a}}\|u\|_{L^{2}\left(\Omega \backslash \bar{\Omega}_{n}\right)}^{2} .
\end{aligned}
$$


Combining (4.6) and (4.7), we get

$$
\operatorname{Re}\left(B_{1}(u, u)+B_{2}(u, u)\right) \geqslant c\|u\|_{H^{1}\left(\Omega \backslash \bar{\Omega}_{n}\right)}^{2},
$$

and so our first goal is achieved.

To complete the proof, we will show that the two remaining terms in (4.5) are positive in their real parts. Actually, this task is straightforward, since

$$
\operatorname{Re} B_{3}(u, u)=2 \gamma\|u\|_{L^{2}(\partial \Omega)}^{2} \geqslant 0,
$$

and the fact that $\operatorname{Re} B_{4}(u, u) \geqslant 0$ is stated in Lemma 3.12 .

To give the finishing touch, we will prove that $f$ is a continuous functional.

Lemma 4.5. The linear functional $f: H^{1}\left(\Omega \backslash \bar{\Omega}_{n}\right) \rightarrow \mathbb{C}$, defined by equations (4.2) and (4.3), is continuous.

Proof. Let $u \in H^{1}\left(\Omega \backslash \bar{\Omega}_{n}\right)$. It holds that

$$
\begin{aligned}
|f(u)| & \leqslant 2\left\|\Phi_{\text {in }}\right\|_{L^{2}(\partial \Omega)}\|u\|_{L^{2}(\partial \Omega)} \\
& \leqslant C\left\|\Phi_{\text {in }}\right\|_{L^{2}(\partial \Omega)}\|u\|_{H^{1}\left(\Omega \backslash \bar{\Omega}_{n}\right)},
\end{aligned}
$$

where we, again, used the Schwarz inequality and the trace theorem.

Finally, we are able to state the main result of this work. We are not content with the existence and uniqueness result but also say something about the continuous dependence of the solution on the boundary data.

Theorem 4.6. The direct weak problem in the strongly scattering region, as introduced in Definition 4.1, has a unique solution $\varphi \in H^{1}\left(\Omega \backslash \bar{\Omega}_{n}\right)$. In addition, the solution depends continuously on the boundary data $\Phi_{\text {in }}$, meaning that

$$
\|\varphi\|_{H^{1}\left(\Omega \backslash \bar{\Omega}_{n}\right)} \leqslant C\left\|\Phi_{\text {in }}\right\|_{L^{2}(\partial \Omega)} .
$$

Proof. As mentioned earlier, the existence and uniqueness result is a straight consequence of the Lax-Milgram Lemma and Lemmas 4.3, 4.4 and 4.5. On the other hand, the continuous dependence follows by using the coercivity of $B$ and inequality (4.8):

$$
\begin{aligned}
\|\varphi\|_{H^{1}\left(\Omega \backslash \bar{\Omega}_{n}\right)}^{2} & \leqslant C|B(\varphi, \varphi)| \\
& =C|f(\varphi)| \\
& \leqslant C^{\prime}\left\|\Phi_{\mathrm{in}}\right\|_{L^{2}(\partial \Omega)}\|\varphi\|_{H^{1}\left(\Omega \backslash \bar{\Omega}_{n}\right)},
\end{aligned}
$$

and so, dividing by $\|\varphi\|_{H^{1}\left(\Omega \backslash \bar{\Omega}_{n}\right)}$, we get the desired result. 


\section{References}

1. S. R. ArRidge, Optical tomography in medical imaging, Inverse Problems 15 (1999), R41.

2. S. R. Arridge And J. C. Hebden, Optical imaging in medicine. II. Modelling and reconstruction, Phys. Med. Biol. 42 (1997), 841-853.

3. K. M. CASE, Linear transport theory (Addison-Wesley, 1967).

4. D. Colton And R. KREss, Integral equation methods in scattering theory (Wiley, 1983).

5. H. Dehghani, S. R. Arridge, M. Schweiger and D. T. Delpy, Optical tomography in the presence of void regions, J. Opt. Soc. Am. 17 (2000), 1659-1670.

6. P. Grisvard, Elliptic problems in non-smooth domains (Pitman, 1985).

7. J. C. Hebden and S. R. Arridge, Optical imaging in medicine. I. Experimental techniques, Phys. Med. Biol. 42 (1997), 825-840.

8. J. Heino AND E. Somersalo, Estimation of optical absorption in anisotropic backround, Inv. Prob., in press.

9. V. Hutson And J. S. Pym, Applications of functional analysis and operator theory (Academic, 1980).

10. M. REED AND B. SIMON, Methods of modern mathematical physics. I. Functional analysis (Academic, 1980).

11. J. R. Ringrose, Compact non-self-adjoint operators (Van Nostrand Reinhold, 1971).

12. W. Rudin, Real and complex analysis (McGraw-Hill, 1987).

13. K. YosidA, Functional analysis (Springer, 1980). 